\title{
Review of: "AudBility: Effectiveness of an online central auditory processing screening program"
}

tayseer abdelrahman

Potential competing interests: The author(s) declared that no potential competing interests exist.

Needs English language revision especially the Limitations section

also it is not limitation that formal tests were not applied or, peripheral cochlear screening was not performed. 